\title{
Magnetic response of a quantum wire of elliptical cross-section in a magnetic field perpendicular to the axis of the wire
}

\author{
V. A. Margulis ${ }^{1}$, V. V. Karpunin ${ }^{2}$, K. I. Mironova ${ }^{1}$ \\ ${ }^{1}$ Ogarev Mordovian State University, Bolshevistskaya 68, Saransk, 340005, Russia \\ ${ }^{2}$ Mordovian State Pedagogical Institute named after M. E. Evseviev, Studencheskaya 11 A, \\ Saransk, 340007, Russia \\ theorphysics@mrsu.ru, karpuninvv@mail.ru
}

PACS 07.55.Jg, 73.21.Hb

DOI 10.17586/2220-8054-2018-9-2-244-251

The magnetic response of a quantum wire of elliptical cross section is investigated. An explicit analytic expression is found for the spectrum and wave functions of an electron in the wire. Using an approach based on finding the classical partition function, an expression is obtained for the magnetic response of the electron gas in the wire. The dependence of the response on the magnitude and direction of the magnetic field is found.

Keywords: magnetic moment, quantum wire.

Received: 6 February 2018

Revised: 12 February 2018

\section{Introduction}

Effects arising in the magnetic field perpendicular to the axis of the wire in electronic systems are due to the fact that the motion in the plane of confinement and perpendicular to it is connected by a magnetic field whose direction is determined by the angle $\theta, \mathbf{B}=B(\sin \theta, \cos \theta)$ [1-4]. The effect of the spin-orbit interaction on the magnetization of low-dimensional systems has been investigated [5-13]. The energy spectrum of electrons in low-dimensional systems has been studied in [14]. As shown in [1,2], in the case of the parabolic confinement potential (well or wire), the problem of finding the spectrum and wave functions of the one-electron Hamiltonian can be solved exactly.

The magnetoresistance of the wire in a magnetic field whose direction is at different angles with the wire axis has been studied experimentally in Refs [15]. It is shown that at different angles the Aharonov-Bohm oscillations are manifested in the magnetoresistance.

The jumps of the magnetic moment for the wire in the form of a thin-walled cylinder were found in [16]. We note that theoretical studies of the magnetic moment of the wire in a magnetic field transverse to the axis are not known to us. In [17], the electron-impurity scattering coefficient of Bloch waves for one dimensional Dirac comb potential is used for calculating the temperature dependence of conductivity within kinetic theory. Nonlinear light absorption and its time evolution at high optical excitation levels in GaSe and InSe layered crystals have been experimentally investigated in [18].

We use an asymmetric parabolic potential $V$ for modeling of a quantum waveguide with elliptical cross-section. In this case, the confinement potential is:

$$
V(x, z)=\frac{m^{*}}{2}\left(\omega_{1}^{2} x^{2}+\omega_{2}^{2} z^{2}\right)
$$

here $\omega_{1}$ and $\omega_{2}$ is effective frequencies of potential, $m^{*}$ is the effective electronic mass. In this model, the characteristic lengths are $l_{i}=\sqrt{\hbar / m^{*} \omega_{i}}$, where $i=1,2$ equal to the semi-axes of the cross-section of the wire. We choose the gauge of the magnetic field in the form $\mathbf{A}=\left(0, B_{z} x-B_{x} z, 0\right)$. The axis of the wire is located along the axis oy. The Hamiltonian describing the one-electron states has the form:

$$
H=\frac{1}{2 m^{*}}\left[p_{x}^{2}+\left(p_{y}-\frac{e}{c} B_{z} x+\frac{e}{c} B_{x} z\right)^{2}+p_{z}^{2}\right]+V(x, z)
$$

The solution of the Schrödinger equation with the Hamiltonian (2) can be written in the form:

$$
\Psi(x, y, z)=\frac{1}{\sqrt{L}} \exp \left(\frac{i}{\hbar} p_{y} y\right) \varphi(x, z),
$$


where $\varphi$ satisfies the equation $\widehat{H} \varphi=E \varphi$, and $L$ is the length of the wire. This $L^{-1 / 2}$ is the normalization constant of the plane wave in the y direction. The Hamiltonian $\widehat{H}$ has the form

$$
\widehat{H}=\frac{1}{2 m^{*}}\left[p_{x}^{2}+\left(p_{y}-\frac{e}{c} B_{z} x+\frac{e}{c} B_{x} z\right)^{2}+p_{z}^{2}\right]+V(x, z)
$$

expression (2) is not identical (4) because $p_{y}$ is eigenvalue of the operator $p_{y}$.

Let us shift the coordinate system $x o z$ along the axis $x$ by $x_{0}$, and along the axis $z$ by $z_{0}$, and then we require the reduction of the linear in the axis $x$ and $z$ terms in $h$. Then we obtain equations for $x_{0}, y_{0}$ :

$$
\left\{\begin{array}{l}
-p_{y} \omega_{z}+m^{*}\left[\left(\omega_{1}^{2}+\omega_{z}^{2}\right) x_{0}-\omega_{x} \omega_{z} z_{0}\right]=0 \\
p_{y} \omega_{x}+m^{*}\left[\left(\omega_{2}^{2}+\omega_{x}^{2}\right) z_{0}-\omega_{x} \omega_{z} x_{0}\right]=0
\end{array}\right.
$$

where $\omega_{x}=|e| B_{x} / m^{*} c, \omega_{z}=|e| B_{z} / m^{*} c, \quad B_{x}=B \sin \theta . \theta$ is the angle defined by the magnetic field and the $\mathrm{x}$ axis.

Solving the system (5), we get relations

$$
x_{0}=\frac{\omega_{z} p_{y}}{M \omega_{1}^{2}} ; \quad z_{0}=-\frac{\omega_{x} p_{y}}{M \omega_{2}^{2}} .
$$

After the shift in the axes $x^{\prime}=x-x_{0}, \quad z^{\prime}=z-z_{0}$, the Hamiltonian $\hat{H}$ takes the form:

$$
\begin{gathered}
\widehat{H}=-\frac{\hbar^{2}}{2 m^{*}} \Delta+\frac{p_{y}^{2}}{2 m^{*}}+\left(\omega_{x} z_{0}-\omega_{x} x_{0}\right) p_{y}+ \\
\frac{m^{*}}{2}\left[\left(\omega_{1}^{2}+\omega_{z}^{2}\right)\left(x^{\prime 2}+x_{0}^{2}\right)+\right. \\
\left.+\left(\omega_{2}^{2}+\omega_{x}^{2}\right)\left(z^{\prime 2}+z_{0}^{2}\right)-2 \omega_{x} \omega_{z}\left(x^{\prime} z^{\prime}+x_{0} z_{0}\right)\right],
\end{gathered}
$$

where $\Delta$ is a two-dimensional Laplace operator.

We rotate the coordinate system by an angle $\alpha$ to get rid of the term $\sim x^{\prime} z^{\prime}$. The angle $\alpha$ satisfies the relation:

$$
\operatorname{tg} 2 \alpha=\frac{2 \omega_{x} \omega_{z}}{\omega_{2}^{2}-\omega_{1}^{2}+\omega_{x}^{2}-\omega_{z}^{2}}
$$

The Hamiltonian $\hat{H}$ takes the form:

$$
\widehat{H}=-\frac{\hbar^{2}}{2 m^{*}} \Delta+\frac{p_{y}^{2}}{2 M}+\frac{m^{*} \Omega_{1}^{2}}{2} x^{2}+\frac{m^{*} \Omega_{2}^{2}}{2} z^{2} .
$$

Characteristic frequencies are:

$$
2 \Omega_{1,2}^{2}=\omega_{1}^{2}+\omega_{2}^{2}+\omega_{c}^{2} \pm \sqrt{\left(\omega_{2}^{2}-\omega_{1}^{2}-\omega_{c}^{2} \cos 2 \theta\right)^{2}+\omega_{c}^{4} \sin ^{2} 2 \theta}
$$

The spectrum $\hat{H}$, as follows from (9), has the form:

$$
\varepsilon=\frac{p_{y}^{2}}{2 M}+\hbar \Omega_{1}\left(n_{1}+\frac{1}{2}\right)+\hbar \Omega_{2}\left(n_{2}+\frac{1}{2}\right), \quad n_{1}, n_{2}=0,1,2 \ldots
$$

where:

$$
M=m^{*}\left(1+\frac{\omega_{x}^{2}}{\omega_{2}^{2}}+\frac{\omega_{z}^{2}}{\omega_{1}^{2}}\right)=m^{*}\left(1+\frac{\omega_{c}^{2}}{\omega_{2}^{2}} \sin ^{2} \theta+\frac{\omega_{c}^{2}}{\omega_{1}^{2}} \cos ^{2} \theta\right)
$$

Eigen functions $\varphi(x, z)$ have the form:

$$
\varphi(x, z)=\Phi_{n_{1}}\left[\left(x-x_{0}\right) \cos \alpha+\left(z-z_{0}\right) \sin \alpha\right] \times \Phi_{n_{2}}\left[\left(x-x_{0}\right) \sin \alpha+\left(z-z_{0}\right) \cos \alpha\right]
$$

where $\Phi_{n_{1}}$ and $\Phi_{n_{2}}$ are the oscillatory functions. $\Phi_{s}(y)=C_{s} \exp \left(-y^{2} / 2 l^{2}\right) H_{s}(y / l), C_{s}$ is the normalization constant, $H_{s}(y / l)$ is Hermite polynomial. 


\section{Magnetic moment}

The purpose of this paper is to find the magnetic moment $\mathbf{M}$ of the wire placed in a perpendicular magnetic field. The calculation of the magnetic moment is based on finding the thermodynamic potential $\Omega$ of the gas in the wire. Then $\mathbf{M}$ is determined by the formula $\mathbf{M}=-(\partial \Omega / \partial \mathbf{B})_{T, V}$, where $T$ is temperature and $V$ is volume.

There are two approaches to finding $\Omega$. One approach is based on the Poisson formula $[19,20]$, and the other [21], is based on finding the classical partition function of states $Z$. Both approaches were used earlier to find the magnetic response of nanostructures [22-24]. In this paper, we use the classical partition function $Z$ for finding the monotonic and oscillating part of the magnetic moment:

$$
Z=\sum_{n_{1}, n_{2}, p_{y}} \exp \left(-\varepsilon_{n_{1}, n_{2}, p_{y}} / T\right)
$$

Using the formula (11), from (14) we get:

$$
Z=\frac{D \sqrt{T}}{4}\left[\sinh \left(\frac{\hbar \Omega_{1}}{2 T}\right) \sinh \left(\frac{\hbar \Omega_{2}}{2 T}\right)\right]^{-1}
$$

where $D=\sqrt{2 \pi M} L /(2 \pi \hbar)$.

Introducing $\zeta=1 / T$, we obtain for $\Omega$ the expression [21]:

$$
\Omega=\frac{1}{2 \pi i} \int_{\gamma-i \infty}^{\gamma+i \infty} \frac{Z(\zeta)}{\zeta^{2}} d \zeta \int_{0}^{\infty} e^{\varepsilon \zeta} \frac{\partial f_{0}}{\partial \varepsilon} d \varepsilon
$$

Here, $f_{0}(\varepsilon)=(\exp ((\varepsilon-\mu) / T)+1)^{-1}$ is the Fermi distribution function $0<\gamma<1 / T$. In (16) the expression for $\Omega$ contain the integral:

$$
\frac{1}{2 \pi i} \int_{\gamma-i \infty}^{\gamma+i \infty} \frac{\exp \varepsilon \zeta d \zeta}{\zeta^{5 / 2} \sinh \beta_{1} \zeta \sinh \beta_{2} \zeta}
$$

where $\beta_{1,2}=\hbar \Omega_{1,2} / 2$. This integral is calculated in the Appendix:

$$
\begin{gathered}
\frac{1}{2 \pi i} \int_{\gamma-i \infty}^{\gamma+i \infty} \frac{\exp (\varepsilon \zeta) d \zeta}{\zeta^{5 / 2} \sinh \beta_{1} \zeta \sinh \beta_{2} \zeta}=\frac{1}{\pi} \int_{0}^{\infty} \frac{\exp (-\varepsilon t) d t}{t^{5 / 2} \sinh \beta_{1} t \sinh \beta_{2} t}+\frac{2}{\pi^{5 / 2}} \sum_{k=1}^{\infty} \frac{(-1)^{k}}{k^{5 / 2}} \times \\
{\left[\beta_{1}^{3 / 2} \frac{\cos \left(k \pi \varepsilon / \beta_{1}+\pi / 4\right)}{\sin \left(\pi k \beta_{2} / \beta_{1}\right)}+\beta_{2}^{3 / 2} \frac{\cos \left(k \pi \varepsilon / \beta_{2}+\pi / 4\right)}{\sin \left(\pi k \beta_{1} / \beta_{2}\right)}\right] .}
\end{gathered}
$$

Replacing in (16) $(\varepsilon-\mu) / T=\xi$, where $\mu$ is the chemical potential of the electronic gas and replacing the value $-\mu / T$ on $-\infty$, we obtain from (16) and (18) the expression for $\Omega=\Omega^{\text {mon }}+\Omega^{o s c}$, where:

$$
\Omega^{m o n}=-\frac{D T}{4} \times \int_{0}^{\infty} \exp (-\mu t)\left[t^{3 / 2} \sin (\pi T t) \sinh \beta_{1} t \sinh \beta_{2} t\right]^{-1} d t .
$$

The oscillating part $\Omega^{o s c}$ has the form of a Fourier series:

$$
\Omega^{o s c}=-\frac{T D}{2 \sqrt{\pi}} \sum_{k=1}^{\infty} \frac{(-1)^{k+1}}{k^{3 / 2}}\left[\frac{\beta_{1}^{1 / 2} \cos \left(\pi \mu k / \beta_{1}+\pi / 4\right)}{\sinh \left(\pi^{2} k T / \beta_{1}\right) \sin \left(\pi k \beta_{2} / \beta_{1}\right)}+\frac{\beta_{2}^{1 / 2} \cos \left(\pi \mu k / \beta_{2}+\pi / 4\right)}{\sinh \left(\pi^{2} k T / \beta_{2}\right) \sin \left(\pi k \beta_{1} / \beta_{2}\right)}\right] .
$$

It is convenient at first calculate the derivatives:

$$
\frac{\partial \Omega_{1,2}}{\partial B}=\frac{e \omega_{c}}{m^{*} c \Omega_{1,2}}\left(1 \pm \frac{\omega_{1}^{2}-\omega_{2}^{2}+\omega_{c}^{2}}{\Omega_{1}^{2}-\Omega_{2}^{2}}\right) .
$$

Differentiating $\Omega^{o s c}$ only rapidly oscillating factors in the field, and taking account of (20), we obtain:

$$
\begin{gathered}
\frac{M^{o s c}}{\mu_{B}^{*}}=-\frac{1}{2} T L \sqrt{M} \mu \omega_{c} \times\left\{\frac{1}{\left(\hbar \Omega_{1}\right)^{5 / 2}}\left(1+\frac{\omega_{1}^{2}-\omega_{2}^{2}+\omega_{c}^{2}}{\Omega_{1}^{2}-\Omega_{2}^{2}}\right) \times\right. \\
\sum_{k=1}^{\infty} \frac{(-1)^{k}}{\sqrt{k}} \frac{\sin \left(\frac{2 \pi k \mu}{\hbar \Omega_{1}}+\frac{\pi}{4}\right)}{\sinh \left(\frac{2 \pi^{2} k T}{\hbar \Omega_{1}}\right) \sin \left(\pi k \frac{\Omega_{2}}{\Omega_{1}}\right)}+\frac{1}{\left(\hbar \Omega_{2}\right)^{5 / 2}}\left(1+\frac{\omega_{1}^{2}-\omega_{2}^{2}+\omega_{c}^{2}}{\Omega_{2}^{2}-\Omega_{1}^{2}}\right) \times
\end{gathered}
$$




$$
\left.\sum_{k=1}^{\infty} \frac{(-1)^{k}}{\sqrt{k}} \frac{\sin \left(\frac{2 \pi k \mu}{\hbar \Omega_{2}}+\frac{\pi}{4}\right)}{\sinh \left(\frac{2 \pi^{2} k T}{\hbar \Omega_{2}}\right) \sin \left(\pi k \frac{\Omega_{1}}{\Omega_{2}}\right)}\right\}
$$

here $\mu_{B}^{*}=e \hbar / 2 m^{*} c$.

In the case $\omega^{2}=\omega_{1}^{2}=\omega_{2}^{2}$, however $\Omega_{1} \neq \Omega_{2}, \Omega_{1}=\sqrt{\omega^{2}+\omega_{c}^{2}}, \Omega_{2}=\omega$. In this connection, for the circular cross section the formula (22) has the form

$$
\begin{gathered}
\frac{M_{o s c}}{\mu_{B}^{*}}=-T L \sqrt{M} \mu \omega_{c} \times\left[\frac{1}{\left(\hbar \sqrt{\omega^{2}+\omega_{c}^{2}}\right)^{5 / 2}} \times\right. \\
\left.\sum_{k=1}^{\infty} \frac{(-1)^{k}}{\sqrt{k}} \frac{\sin \left(\left(2 \pi k \mu / \hbar \sqrt{\omega^{2}+\omega_{c}^{2}}\right)+\pi / 4\right)}{\sinh \left(2 \pi^{2} k T / \hbar \sqrt{\omega^{2}+\omega_{c}^{2}}\right) \sin \left(\pi k \omega / \sqrt{\omega^{2}+\omega_{c}^{2}}\right)}\right] .
\end{gathered}
$$

It can be noted that passage to the limit to a wire of circular cross section $\omega_{c} \neq 0$ is possible, since in this case $\Omega_{1} \neq \Omega_{2}$. In the case, when $\omega_{c} \rightarrow 0$, such passage to the limit is impossible. The reason for this is related to the fact that the calculation of the magnetic moment in the work is based on finding the partition function $Z$ (15). The oscillating part is formed by the sum of the residues at the poles of the first order at the points $z_{k}$.

$$
\operatorname{res}\left[\frac{\exp (\varepsilon z)}{z^{5 / 2} \sinh \beta_{1} z \sinh \beta_{2} z}, z_{k}\right] \text {. }
$$

If $\Omega_{1}=\Omega_{2}, \omega_{c}=0$, the poles become of the second order:

$$
\operatorname{res}\left[\frac{\exp (\varepsilon z)}{z^{5 / 2} \sinh ^{2} \beta z}, z_{k}\right]=\lim _{z \rightarrow z_{k}}\left[\frac{\exp (\varepsilon z)\left(z-z_{k}\right)^{2}}{z^{5 / 2} \sinh ^{2} \beta z}\right] .
$$

Because of this circumstance, using formula (A.7) and hence (23) in this case cannot be found as $\lim _{\omega_{c} \rightarrow 0} M_{o s c}$ because poles are changes.

The dependence of the magnetic response on the magnetic field is shown in Figs. $1-4$. Let us now consider the limit of the potential $\Omega$ in the case of weak fields: $\omega_{c} \rightarrow 0, \mu \gg \hbar \Omega_{1,2}, \hbar \Omega_{1,2} / T \gg 1$. We note that in this limit, the oscillating part $\Omega^{o s c}$ consists of rapidly oscillating terms of the series (20) and their contribution to $\Omega$ tends to zero. To evaluate $\Omega^{\text {mon }}$, we use (16). Then, as shown in [25] at low temperatures, the expression is given by:

$$
-\Omega=z(\mu)+\frac{\pi^{2} T^{2}}{6} z^{\prime \prime}(\mu)
$$

where

$$
z(\varepsilon)=\frac{1}{2 \pi i} \int_{\gamma-i \infty}^{\gamma+i \infty} e^{\varepsilon \xi} Z(\xi) \frac{d \xi}{\xi^{2}}
$$

here $Z(\xi)$ is determined by the formula (15). With the constraint $\hbar \Omega_{i} / T \gg 1$ from (15) follows the estimate

$$
Z(\xi) \simeq \frac{D}{\sqrt{\xi}} \exp \left[-\frac{\hbar\left(\Omega_{1}+\Omega_{2}\right)}{T}\right] .
$$

We substitute (28) in (27) and use the formula [26]:

$$
\frac{1}{2 \pi i} \int_{\gamma-i \infty}^{\gamma+i \infty} t^{\gamma-1} e^{-p t} d t=\left\{\begin{array}{l}
0, \quad p>0, \\
1 /\left((-p)^{\gamma} \Gamma(1-\chi)\right), \quad p<0, \text { at } \gamma>0, \operatorname{Re} \chi<1
\end{array}\right.
$$

Then

$$
z(\varepsilon)=\frac{D}{\Gamma(5 / 2)}\left(\varepsilon-\frac{\hbar \Omega_{1}+\hbar \Omega_{2}}{2}\right)^{3 / 2},
$$

where $\Gamma(\alpha)$ - Euler function, $\Gamma(5 / 2)=3 \sqrt{\pi} / 4$.

The second derivative $z(\varepsilon)$ has the form:

$$
z^{\prime \prime}(\varepsilon)=\frac{D}{\sqrt{\pi}} \frac{1}{\sqrt{\varepsilon-\frac{\hbar\left(\Omega_{1}+\Omega_{2}\right)}{2}}},
$$




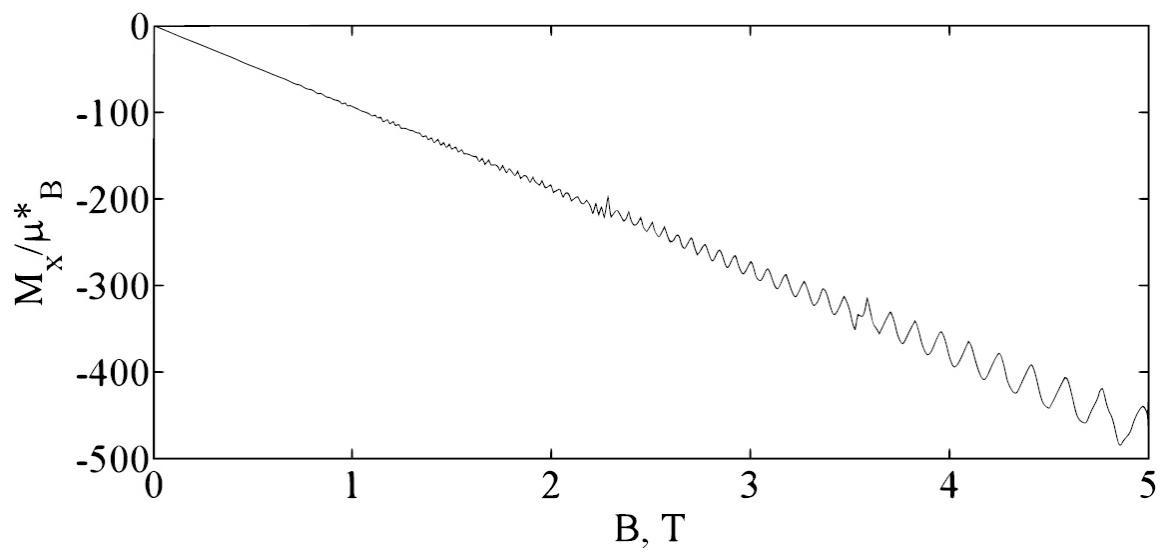

FIG. 1. Dependence of the magnetic response of a quantum wire on the magnitude of the magnetic field for the angle $\theta=\pi / 8.324$, the effective frequencies of the confinement potential are $\omega_{1}=2.177 \times 10^{12} c^{-1} ; \omega_{2}=3.353 \times 10^{12} c^{-1}$. The temperature $T$ is $7 \mathrm{~K}$

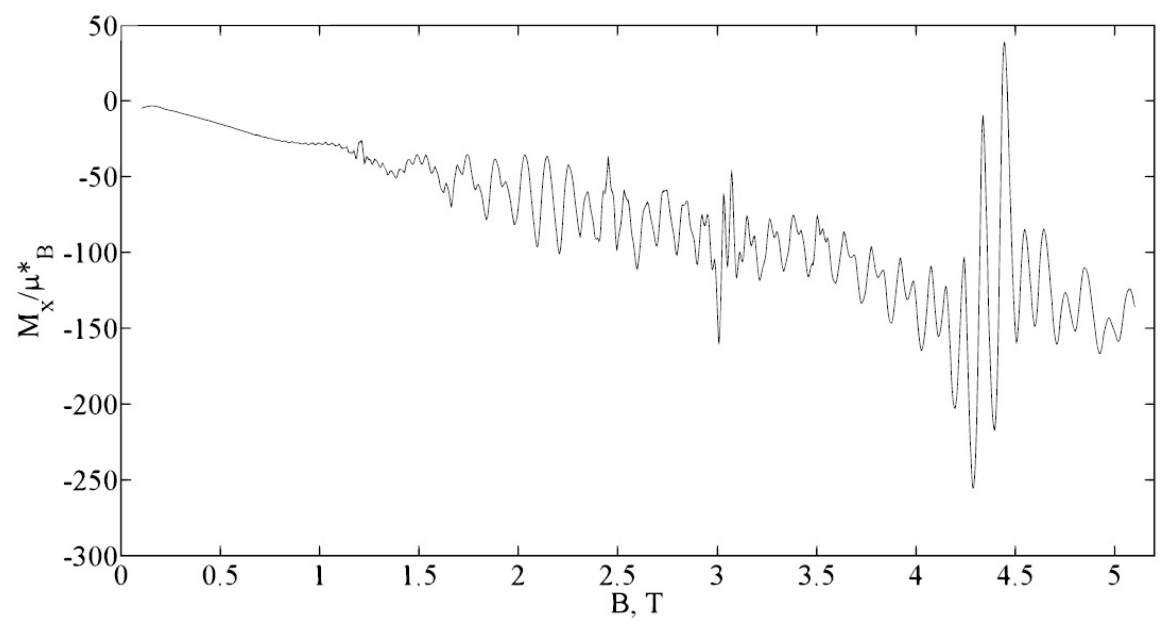

FIG. 2. Dependence of the magnetic response of a quantum wire on the magnitude of the magnetic field for the angle $\theta=\pi / 9.524$, the effective frequencies of the confinement potential are $\omega_{1}=3.177 \times 10^{12} c^{-1} ; \omega_{2}=6.353 \times 10^{12} c^{-1}$. The temperature $T$ is $3 \mathrm{~K}$

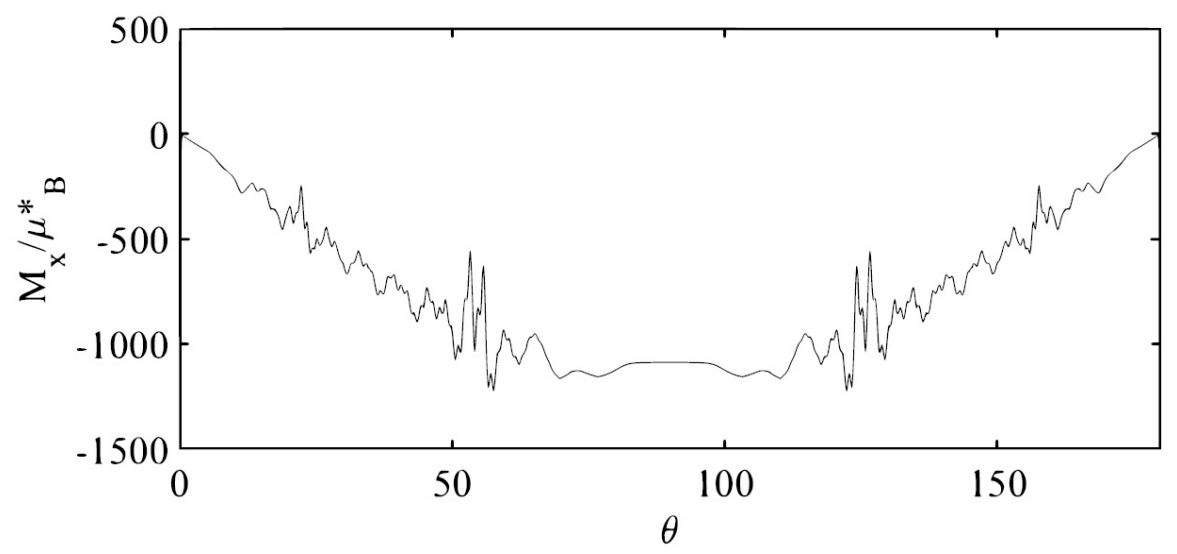

FIG. 3. The dependence of the magnetic response of a quantum wire on the direction of the magnetic field (in the interval from 0 to 180 ), the value of which is $B=1.52 \mathrm{Tl}$, at effective frequencies of the confinement potential are $\omega_{1}=2.177 \times 10^{12} c^{-1} ; \omega_{2}=3.353 \times 10^{12} c^{-1}$. The temperature $T$ is $2 \mathrm{~K}$ 


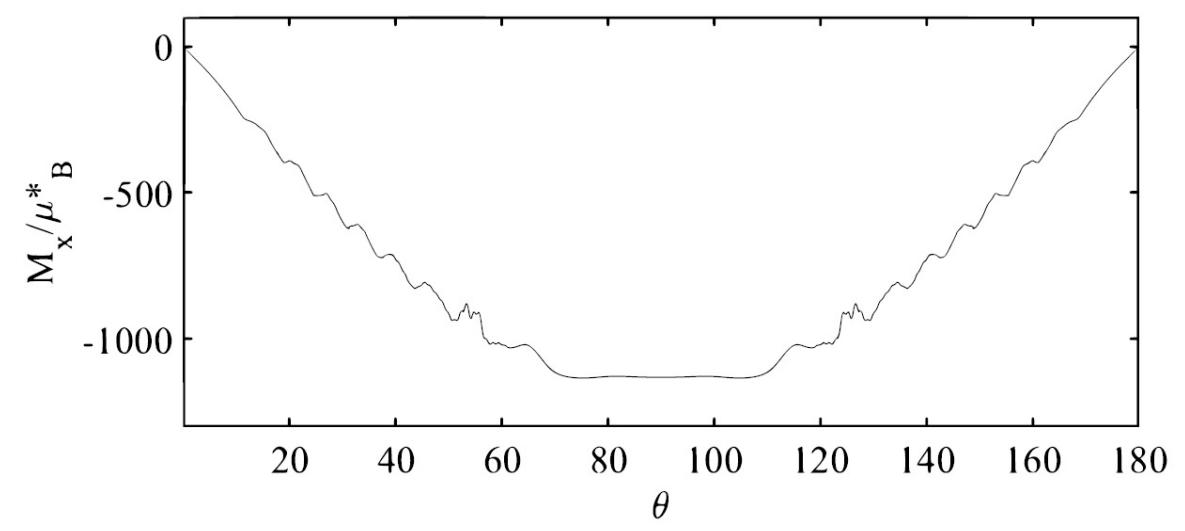

FIG. 4. The dependence of the magnetic response of a quantum wire on the direction of the magnetic field (in the interval from 0 to 180 ), the value of which is $B=1.52 \mathrm{Tl}$, at effective frequencies of the confinement potential are $\omega_{1}=2.177 \times 10^{12} c^{-1} ; \omega_{2}=3.353 \times 10^{12} c^{-1}$. The temperature $T$ is $5 \mathrm{~K}$

Then, for $\Omega^{\text {mon }}$, we obtain:

$$
-\Omega^{\text {mon }}(\mu)=\frac{4 D}{3 \sqrt{\pi}}\left(\mu-\frac{\hbar \Omega_{1}+\hbar \Omega_{2}}{2}\right)^{3 / 2}+\frac{D}{\sqrt{\pi}} \frac{\pi^{2} T^{2}}{6} \frac{1}{\sqrt{\mu-\frac{\hbar \Omega_{1}+\hbar \Omega_{2}}{2}}} .
$$

The second term in (32) is small in comparison with the first $\sim O\left(T^{2} / \mu^{2}\right)$, so this can be neglected.

For the estimate $\Omega^{m o n}$, we confine ourselves to the case $\omega_{1}=\omega_{2}$. Then, $D \simeq D_{0} \sqrt{1+\omega_{c}^{2} / \omega^{2}}$, where $D_{0}=D(B=0)$. Frequencies $\Omega_{1}=\omega, \Omega_{2}=\sqrt{\omega^{2}+\omega_{c}^{2}}$.

$$
-\Omega^{\text {mon }}(\mu)=\frac{4 D}{3 \sqrt{\pi}}\left(\mu-\hbar \omega-\frac{\hbar \omega_{c}^{2}}{4 \omega}\right)^{3 / 2}\left(1+\frac{\omega_{c}^{2}}{2 \omega^{2}}\right) .
$$

From (33) we obtain the estimate:

$$
-\frac{1}{\mu_{B}^{*}} \frac{\partial \Omega^{\text {mon }}}{\partial B} \simeq \frac{8 D_{0}}{3 \sqrt{\pi}} \frac{\omega_{c}}{\hbar \omega^{2}}(\mu-\hbar \omega)^{3 / 2}-\frac{2 D_{0} \omega_{c}}{\sqrt{\pi} \omega}(\mu-\hbar \omega)^{1 / 2} .
$$

In (34) to within $\hbar \omega / \mu$ we can confine ourselves to the first term. Then for the magnetic moment, we obtain:

$$
\frac{M^{m o n}}{\mu_{B}^{*}}=\frac{8 D_{0}}{3 \sqrt{\pi}} \frac{\omega_{c}}{\hbar \omega^{2}}(\mu-\hbar \omega)^{3 / 2} .
$$

From (35), one can estimate the magnetic susceptibility of an electron gas in a wire $\chi=-(1 / V) \partial^{2} \Omega / \partial B^{2}$. Here, the normalization volume $V=\hbar L / m^{*} \sqrt{\omega_{1} \omega_{2}}$. Then, for susceptibility $\chi$, we obtain the following expression:

$$
\chi=\frac{4 D_{0}}{3 \sqrt{\pi} L}\left(\frac{e^{2}}{m^{*} c^{2}}\right) \frac{(\mu-\hbar \omega)^{3 / 2}}{\hbar \omega} .
$$

\section{Conclusion}

As can be seen from formula (35) and (Fig. 1), the main dependence is linear on the magnetic field. Peaks grow linearly with increasing magnetic field.

We consider the wire with an elliptical section, not a circular section, since the latter type of section is a special case. In particular, in the case of a circular cross section, the value $M$ entering the spectrum does not depend on the angle of field direction $\theta, M=m^{*}\left(1+\omega_{c}^{2} / \omega^{2}\right), \omega^{2}=\omega_{1}^{2}=\omega_{2}^{2}$.

It follows from the formula (36) that the electron gas in the wire is paramagnetic $\chi>0$ and the cause of this is the dependence $M(B)$. Obviously, the expressions (20) and (22) for $\Omega^{o s c}$ and $M^{o s c}$ lose their meaning for those values of the field $B$ for which the ratio $\Omega_{1} / \Omega_{2}$ or $\Omega_{2} / \Omega_{1}$ is equal to an integer (the commensurability condition). The question of the convergence of Fourier series of the type entering into (20) and (22) is considered in detail in [2], where it is shown that with probability one the series converge to an analytic function and, therefore, they can be differentiated term by term in components fields at all points where the commensurability conditions are not satisfied. 
In Fig. 1-4, we plotted the dependence of the magnetic moment on the magnitude and direction of the field at typical values for the spectrum parameters. It is important to note that oscillatory dependencies arise not only on the graph $M(B)$, but also $M(\theta)$. In both cases, the oscillations are not periodic.

\section{Appendix}

We introduce the notation:

$$
J=\frac{1}{2 \pi i} \int_{\gamma-i \infty}^{\gamma+i \infty} \frac{\zeta^{3 / 2} e^{\varepsilon \zeta} d \zeta}{\sinh \beta_{1} \zeta \sinh \beta_{2} \zeta} .
$$

For the calculation $J$, we extend the integrand to the complex plane with a cut along the negative part of the real line. Consider a contour $\Gamma$ consisting of both $C_{R}, C_{\rho}$ segments and a segment $[\rho, R] \mathrm{T}[R, \rho]$, and $[\gamma-i R, \gamma-i R]$ (Fig.5).

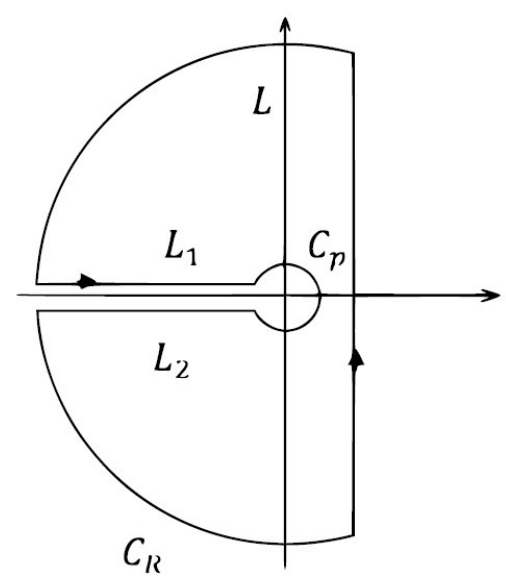

FIG. 5. Contour $\Gamma$

The integrand in $J$ is

$$
\frac{d^{4}}{d \varepsilon^{4}}\left(\frac{e^{\varepsilon \zeta}}{\zeta^{5 / 2} \sinh \beta_{1} \zeta \sinh \beta_{2} \zeta}\right) .
$$

This function has simple poles at points $z_{k}=i \pi k / \beta_{1}$, and $z_{k}=i \pi k / \beta_{2}$, where $k= \pm 1, \pm 2, \ldots$. It is easy to show that the integrals over $C_{R}, C_{\rho}$ tend to zero for $R \rightarrow \infty$ and $\rho \rightarrow 0$, respectively. The sum of the integrals over the upper and lower sides of the cut in the same range gives:

$$
\frac{1}{\pi} \int_{0}^{\infty} \frac{t^{3 / 2} e^{-\varepsilon t} d t}{\sinh \beta_{1} t \sinh \beta_{2} z}
$$

We take into account that:

$$
\frac{1}{2 \pi i} \oint_{\Gamma} \frac{\zeta^{3 / 2} e^{\varepsilon \zeta} d \zeta}{\sinh \beta_{1} \zeta \sinh \beta_{2} \zeta}=\sum_{k=-\infty}^{\infty} \operatorname{res}\left[\frac{z^{3 / 2} e^{\varepsilon z}}{\sinh \beta_{1} z \sinh \beta_{2} z}, z_{k}\right] .
$$

Then we get the relation:

$$
\frac{1}{2 \pi i} \int_{\gamma-i \infty}^{\gamma+i \infty} \frac{\zeta^{3 / 2} e^{\varepsilon \zeta} d \zeta}{\sinh \beta_{1} \zeta \sinh \beta_{2} \zeta}=\frac{1}{\pi} \int_{0}^{\infty} \frac{t^{3 / 2} e^{-\varepsilon t} d t}{\sinh \beta_{1} t \sinh \beta_{2} t}+\sum_{k=-\infty}^{\infty} \operatorname{res}\left[\frac{z^{3 / 2} e^{\varepsilon z}}{\sinh \beta_{1} z \sinh \beta_{2} z}, z_{k}\right] .
$$

We note that $J(\varepsilon)$ is the inverse Laplace transform of the integrand. From the theorem on the differentiation of the integral and the image to the parameter $\varepsilon$, it follows that:

$$
\frac{1}{2 \pi i} \int_{\gamma-i \infty}^{\gamma+i \infty} \frac{e^{\varepsilon \zeta} d \zeta}{\zeta^{5 / 2} \sinh \beta_{1} \zeta \sinh \beta_{2} \zeta}=\frac{1}{\pi} \int_{0}^{\infty} \frac{e^{-\varepsilon t} d t}{t^{5 / 2} \sinh \beta_{1} t \sinh \beta_{2} t}+
$$




$$
+\sum_{k=-\infty}^{\infty} \operatorname{res}\left[\frac{e^{\varepsilon z}}{z^{5 / 2} \sinh \beta_{1} z \sinh \beta_{2} z}, z_{k}\right] .
$$

Summing up the residues in (A.6), we obtain:

$$
\begin{gathered}
\frac{1}{2 \pi i} \int_{\gamma-i \infty}^{\gamma+i \infty} \frac{e^{\varepsilon \zeta} d \zeta}{\zeta^{5 / 2} \sinh \beta_{1} \zeta \sinh \beta_{2} \zeta}=\frac{1}{\pi} \int_{0}^{\infty} \frac{e^{-\varepsilon t} d t}{t^{5 / 2} \sinh \beta_{1} t \sinh \beta_{2} t}+ \\
+\frac{2}{\pi^{5 / 2}} \sum_{k=1}^{\infty} \frac{(-1)^{k}}{k^{5 / 2}}\left[\frac{\beta_{1}^{3 / 2} \cos \left(\pi k \varepsilon / \beta_{1}+\pi / 4\right)}{\sin \left(\pi k \beta_{2} / \beta_{1}\right)}+\frac{\beta_{2}^{3 / 2} \cos \left(\pi k \varepsilon / \beta_{2}+\pi / 4\right)}{\sin \left(\pi k \beta_{1} / \beta_{2}\right)}\right] .
\end{gathered}
$$

\section{Acknowledgment}

The work is supported by the Russian Foundation of Basic Research (project 16-02-00475).

\section{References}

[1] Merlin R. Subband-Landau-level coupling in tilted magnetic fields: Exact results for parabolic wells. Sol. State Commun., 1987, 64, P. 99-101.

[2] Geyler V.A., Margulis V.A. Specific heat of quasi-two-dimensional systems in a magnetic field. Phys. Rev. B, 1997, 55, P. $2543-2548$.

[3] Bychkov Yu.A., Mel'nikov V.I., Rashba E.I. Effect of spin-orbit coupling on the energy spectrum of a 2D electron system in a tilted magnetic field. Sov. JETP, 1990, 71, P. 401-405.

[4] Drichko I.L., Smirnov I.Yu., Suslov A.V., Mironov O.A., Leadley D.R. Magnetoresistivity and acoustoelectronic effects in a tilted magnetic field in $\mathrm{p}-\mathrm{Si} / \mathrm{SiGe} / \mathrm{Si}$ structures with an anisotropic g factor. JETP, 2010, 111, P. 495-502.

[5] Herzog F., Heedt S., Goerke S., Ibrahim A., Rupprecht B., Heyn Ch., Hardtdegen H., Schpers Th., Wilde M.A. and Grundler D. Confinement and inhomogeneous broadening effects in the quantum oscillatory magnetization of quantum dot ensembles. J. Phys.: Condens. Matter, 2016, 28, P. 045301.

[6] Wilde M.A. and Grundler D. Alternative method for the quantitative determination of Rashba-and Dresselhaus spin-orbit interaction using the magnetization. New Journal of Physics, 2013, 15, P. 115013.

[7] Wilde M. A., Rupprecht B., Herzog F., Ibrahim A., and Grundler D. Spin-orbit interaction in the magnetization of two-dimensional electron systems. Phys. Status Solidi B, 2014, 251, P. 1710-1724.

[8] Rupprecht B., Heedt S., Hardtdegen H., Schapers Th., Heyn Ch., Wilde M.A., and Grundler D. Frequency anomaly in the Rashba-effect induced magnetization oscillations of a high-mobility two-dimensional electron system. Phys. Rev. B, 2013, 87, P. 035307.

[9] Rupprecht B., Krenner W., Wurstbauer U., Heyn Ch., Windisch T., Wilde M.A., Wegscheider W., and Grundler D. Magnetism in a Mn modulation-doped InAs/InGaAs heterostructure with a two-dimensional hole system. J.Appl.Phys., 2010, 107, P. 093711.

[10] Wilde M.A., Schwarz M.P., Heyn Ch., Heitmann D., and Grundler D. Experimental evidence of the ideal de Haas-van Alphen effect in a two-dimensional system. Phys. Rev. B, 2006, 73, P. 125325.

[11] Ruhe N., Springborn J.I., and Heyn Ch. Simultaneous measurement of the de Haas-van Alphen and the Shubnikov-de Haas effect in a two-dimensional electron system. Phys. Rev. B, 2006, 74, P. 235326.

[12] Wilde M. A., Rhode M., Heyn Ch., Heitmann D., and Grundler D. Direct measurements of the spin and valley splittings in the magnetization of a Si/SiGe quantum well in tilted magnetic fields. Phys. Rev. B, 2005, 72, P. 165429.

[13] Wilde M.A., Springborn J.I., Heyn Ch., Heitmann D., Grundler D. Magnetization of GaAs quantum wires with quasi one-dimensional electron systems. Physica E, 2004, 22, P. 729-732.

[14] Apalkov V.M. and Portnoi M.E. Tuning gaps and phases of a two-subband system in a quantizing magnetic field. Phys. Rev. B, 2002, 65, P. 125310.

[15] Gitsu D.V., Huber T.E., Konopko L.A., and Nikolaeva A.A. Aharonov-Bohm Oscillations in Bi Nanowires. AIP Conference Proceedings, 2006, 850, P. 1409-1410.

[16] Tsindlekht M.I., Genkin V.M., Felner I., Zeides F., Katz N., Gazi S., Chromik S., Dobrovolskiy O.V., Sachser R., Huth M. Magnetic moment jumps in flat and nanopatterned $\mathrm{Nb}$ thin-walled cylinders. Physica C: Superconductivity and its Applications, 2017, 533, P. 101104.

[17] Botman S.A., Leble S.B. Electrical conductivity model for quasionedimensional structures. Nanosystems: Physics, Chemistry, Mathematics, 2017, 8(2), P. 231-235.

[18] Kyazimzade A.G., Salmanov V.M., Huseynov A.G., Mamedov R.M., Salmanova A.A., Ahmedova F.Sh. Nonlinear optical and quantadimensional effects in monoselenide of gallium and indium. Nanosystems: Physics, Chemistry, Mathematics, 2017, 8(5), P. 654-660.

[19] Landau L.D., Lifshitz E.M. Statistical Physics, Third Edition, Part 1: Volume 5 (Course of Theoretical Physics, Volume 5), ButterworthHeinemann; 3 edition, January 15, 1980, 544 p.

[20] Shoenberg D. Magnetic Oscillations in Metals. Cambridge University Press, 1984, 570 p.

[21] Perelomov A.M. Generalized Coherent States and their Applications, Springer-Verlag Berlin Heidelberg, 1986, 320 p.

[22] Geyler V.A., Margulis V.A., Nesmelov A.G., Chuchaev I.I. Magnetic Susceptibility of a Quasi-Two-Dimensional System in a Tilted Magnetic Field. Phys. Sol. St., 1994, 36, P. 1090-1094.

[23] Margulis V.A., Mironov V.A. Magnetic moment of a 2D electron gas with spin-orbit interaction. JETP, 2009, 108, P. 656-660.

[24] Margulis V.A., Mironov V.A. Magnetic moment of the Volcano ring. Phys. Sol. St., 2008, 50, P. 152-158.

[25] Margulis V.A. Magnetization and polarization of the electron gas in multiferroics. Low Temp. Phys., 2014, 40, P. $363-366$.

[26] Prudnikov A.P., Brychkov Yu.A., Marichev O.I. Integrals and series, Elementary Functions, N.Y.: Gordon and Breach, 1986,800 p. 